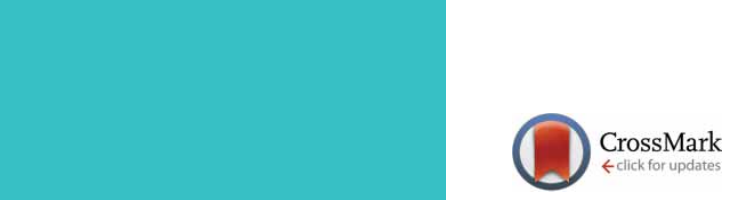

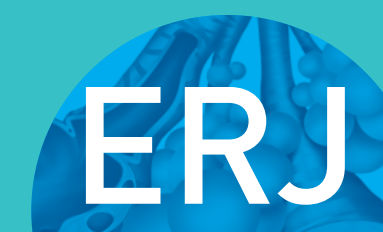

open research
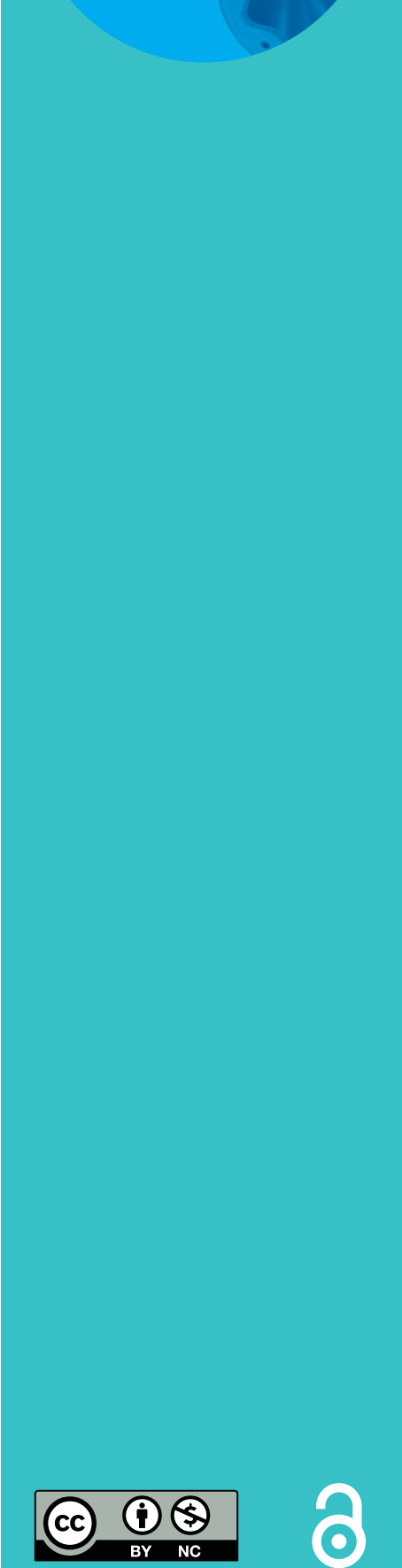

\section{Comparison of intrapleural use of urokinase and tissue plasminogen activator/DNAse in pleural infection}

\author{
Benoît Bédat ${ }^{1}$, Jérôme Plojoux ${ }^{2}$, Jade Noel ${ }^{1}$, Anna Morel ${ }^{1}$, Jonathan Worley ${ }^{1}$, \\ Frédéric Triponez ${ }^{1}$ and Wolfram Karenovics (i) ${ }^{1}$
}

Affiliations: ${ }^{1}$ Thoracic and Endocrine Surgery, University Hospitals of Geneva and Faculty of Medicine, Geneva, Switzerland. 'Division of Pneumology, University Hospitals of Geneva and Faculty of Medicine, Geneva, Switzerland.

Correspondence: Benoît Bédat, Unit of Thoracic and Endocrine Surgery, University Hospitals of Geneva, Rue Gabriel-Perret-Gentil 4, Geneva 1211, Switzerland. E-mail: benoit.bedatahcuge.ch

ABSTRACT Fibrinolysis can be used to improve fluid drainage in pleural infection. Treatment with either urokinase or tissue plasminogen activator (t-PA) in association with DNAse via a chest tube has been effective at reducing the need for surgery. This study is the first to compare the efficacy of these two treatments.

We performed a single-centre, controlled, prospective cohort study. All individuals with pleural infection admitted to our hospital between January 2014 and December 2017 who were treated with antibiotics, a chest tube and fibrinolysis were included in this study. The rate of additional procedure requirements (additional chest tube or surgery) after initial fibrinolysis, complications, costs, and radiological and biological outcomes were analysed.

Among the 93 patients included in this study, 34\% required additional procedures after an initial fibrinolysis, including $21 \%$ who received an additional chest tube and $13 \%$ who underwent thoracoscopy. The need for additional procedures arose due to presence of multiple pleural collections $(p=0.01)$ and was associated with the use of large-bore drain $(\mathrm{p}=0.01)$. The success rate of fibrinolysis was not significantly different between urokinase and t-PA/DNAse $(p=0.35)$. The differences in drainage duration and in length of hospital stay were not significant either $(\mathrm{p}=0.05$ and $\mathrm{p}=0.12$, respectively). Treatment with $\mathrm{t}-\mathrm{PA} / \mathrm{DNAse}$ was cheaper $(p=0.04)$ but was associated with a higher rate of haemothorax $(p=0.002)$.

In conclusion, treatment with urokinase is safer and equally effective when compared with treatment with t-PA/DNAse.

@ERSpublications

In pleural infection, the efficacy of pleural drainage is comparable between high doses of urokinase and tissue plasminogen activator/DNAse http://bit.ly/2lHuxat

Cite this article as: Bédat B, Plojoux J, Noel J, et al. Comparison of intrapleural use of urokinase and tissue plasminogen activator/DNAse in pleural infection. ERJ Open Res 2019; 5: 00084-2019 [https:// doi.org/10.1183/23120541.00084-2019].

This article has supplementary material available from openres.ersjournals.com.

Received: 01 April 2019 | Accepted after revision: 09 July 2019

Copyright $\odot$ ERS 2019. This article is open access and distributed under the terms of the Creative Commons Attribution Non-Commercial Licence 4.0. 


\section{Introduction}

Pleural infection occurs most commonly from adjacent pneumonia. It is an important cause of mortality and morbidity, with an increasing incidence in recent times $[1,2]$. In the USA, pleural infection affects $\sim 32000$ patients per year, with a mortality rate of $10-20 \%[1,3]$. Some consensus guidelines exist for the management of pleural infection, which involves the use of antibiotics and tube drainage $[4,5]$. According to the recommendations, fibrinolytic therapy can be useful but should not be used routinely [4].

Intrapleural injection of fibrinolytics through a chest tube improves fluid drainage. However, the efficacy of fluid drainage differs according to the type of fibrinolytic used. A recent meta-analysis of randomised controlled trials (RCTs) compared fibrinolytics and a placebo treatment [6]. Although this meta-analysis showed a heterogeneity of treatment effects and possible publication bias, it reported that urokinase alone is effective at reducing the rate of surgical referral, unlike tissue plasminogen activator (t-PA) and streptokinase. In another RCT, ALEMÁn et al. [7] demonstrated that there is a higher proportion of clinical and radiological improvements with urokinase compared with t-PA in a subgroup of patients with complicated parapneumonic effusions. In the Multicenter Intrapleural Sepsis Trial (MIST2), treatment with DNAse in association with t-PA improved fluid drainage, shortened lengths of hospital stays and reduced referral for surgery compared to either placebo, t-PA or DNAse alone [8]. Therefore, urokinase and $\mathrm{t}-\mathrm{PA} / \mathrm{DNAse}$ treatments seem to improve clinical outcome in pleural infection.

The aim of this study was to compare the efficacy of high-concentration urokinase therapy with that of t-PA/DNAse therapy in pleural infection and to our knowledge, it is the first study to do so.

\section{Methods}

\section{Patients}

We performed a controlled, prospective cohort study with patients who were hospitalised for pleural effusion or empyema between January 2014 and December 2017 at the University Hospitals of Geneva, Switzerland. All individuals who were treated with antibiotics, a chest tube and fibrinolysis were included in this study. Patients $<18$ years of age or those treated with surgery before fibrinolysis were excluded from the analysis.

The local ethics committee approved this study (referral number 2018-01582). Antibiotic use and chest drainage management were performed in accordance with the guidelines of the British Thoracic Society Pleural Disease Guideline Group [5]. Intrapleural fibrinolysis was used as per conventional institutional practices. Until November 2016, all patients with pleural infection were treated with intrapleural urokinase at a dosage of 250000 units in $30 \mathrm{~mL}$ of $0.9 \% \mathrm{NaCl}$ twice a day for 5 days, according to the protocol developed in our institution. Since December 2016, according to an institutional decision, all patients were treated with intrapleural t-PA/DNAse, with $10 \mathrm{mg}$ t-PA and $5 \mathrm{mg}$ DNAse injected together in $30 \mathrm{~mL}$ of $0.9 \% \mathrm{NaCl}$ twice a day for 3 days. After injection of the fibrinolytics, the chest drainage procedure was identical for both groups: the chest tube was rinsed with $20 \mathrm{~mL}$ of $0.9 \% \mathrm{NaCl}$, closed for $3 \mathrm{~h}$ and then opened in a water-seal system with a suction of $-20 \mathrm{cmH}_{2} \mathrm{O}$.

\section{Measurements and outcomes}

Patient data were obtained prospectively. All data were analysed with respect to patient demographics, pleural fluid findings, radiological findings, type of fibrinolysis, drainage procedure and clinical outcome.

Failure of fibrinolysis is commonly used to assess its effectiveness and is defined as the need for surgical referral [6]. However, this definition may overestimate the effect of fibrinolysis. To compare the efficacy of urokinase treatment with that of t-PA/DNAse treatment, our primary endpoint was the need for an additional procedure after an initial fibrinolysis, determined by the need for an additional chest tube or surgery. In cases of persistent pleural infection despite fibrinolysis, an additional drain with imaging guidance was the first-line procedure, except in severe sepsis or inaccessible collections to drain, or according to the preference of the patient to whom the surgery was proposed.

Secondary endpoints included the length of hospital stay; rate of complications associated with fibrinolysis; duration of drainage; change in the area of pleural opacity, measured on chest radiographs as the percentage of area occupied by effusion before fibrinolysis (baseline) minus the area measured on days 3 and 7 (figure S1); change in serum C-reactive protein (CRP) and leukocyte levels from baseline to days 3 and 7; and costs of fibrinolysis.

\section{Statistical analysis}

All analyses were planned prior to any data collection. The study was completed once a comparable number of patients were included in each group. 
For patient characteristics, Chi-squared or Fisher's exact tests were used to analyse categorical variables. A t-test or Mann-Whitney U-test was used to analyse continuous variables.

For the primary outcome, a subgroup that included patients with surgical referral was analysed. The primary outcome was analysed using logistic regression models, with an assessment of the log-linearity and goodness of fit. For the multivariate analysis, all clinically relevant independent variables were included. A stepwise logistic regression analysis with backward elimination was used.

For the secondary outcomes, Chi-squared or Fisher's exact tests were used to analyse categorical variables. A t-test or Mann-Whitney U-test was used to analyse continuous variables. Data on chest radiography and inflammatory markers obtained after additional drainage or surgery were considered as missing values. Analyses were performed using Stata software, version 14 (StataCorp LLC, College Station, TX, USA).

\section{Results}

\section{Patients}

In total, 99 patients were hospitalised for pleural infection during the study period. Six patients had received fibrinolysis after surgery and were excluded from the study. Of the 93 participants that were included in the analysis: 52 received urokinase and 41 received t-PA/DNAse (table 1). The mean $\pm S D$ patient age was $61.5 \pm 17.2$ years and $17 \%$ of patients had lung cancer associated with empyema. Pleural fluid analyses of both groups were similar and $35 \%$ of patients tested positive for bacterial culture. The majority of patients (92\%) underwent computed tomography (CT) scanning before drainage, which revealed that $70 \%$ of patients had more than one pleural collection, with $2.5 \pm 1.4$ pleural collections per patient on average. In cases with multiple collections, the collections were isolated from each other in $64 \%$ of patients. There were significant differences in the technical procedure used for drainage and tube size between groups (table 1). Image-guided drainage was used in $76 \%$ of the cases, more often in the t-PA/ DNAse group than in the urokinase group $(\mathrm{p}=0.02)$. However, in cases of multiple collections, image-guided drainage was used in $83 \%$ of patients and did not differ between the two groups $(p=0.26)$. There was no association between the presence of isolated collections and the choice of the drainage procedure $(\mathrm{p}=1.0)$. The median drain-tube size was 12 French (range $8-24)$ and the use of a smaller tube was strongly associated with image-guided drainage $(\mathrm{p}<0.001)$. The median duration between hospitalisation and drainage was 3 days (interquartile range (IQR) 1-8 days), and that between drainage and the beginning of fibrinolysis was 1 day (IQR $0-2$ days).

\section{TABLE 1 Patient characteristics}

$\begin{array}{lcccc}\text { Characteristics } & \text { Overall } & \text { Urokinase } & \text { t-PA/DNAse } & \text { p-value } \\ \text { Patients } & 93 & 52 & 41 & \\ \text { Age years } & 61.5 \pm 17.2 & 61.4 \pm 17.6 & 61.6 \pm 17 & 0.95 \\ \text { Males } & 63(68 \%) & 39(75 \%) & 24(59 \%) & 0.09 \\ \text { Right-sided effusion } & 55(59 \%) & 28(54 \%) & 27(66 \%) & 0.24 \\ \text { Presence of lung cancer } & 16(17 \%) & 7(13 \%) & 9(22 \%) & 0.28 \\ \text { Pleural fluid analyses } & 93(100 \%) & & & \\ \quad \text { Positive bacterial culture } & 33(35 \%) & 16(31 \%) & 17(41 \%) & 0.28 \\ \text { pH } & 7 \pm 0.4 & 7 \pm 0.2 & 6.9 \pm 0.4 & 0.63 \\ \text { Glucose mmol-L-1 } & 3.5 \pm 8.1 & 3.9 \pm 9.9 & 2.8 \pm 4.4 & 0.59 \\ \quad \text { Lactate dehydrogenase IU.L-1 } & 2217 \pm 2676 & 2447 \pm 3171 & 1912 \pm 1831 & 0.38 \\ \text { Radiological findings } & & & & \\ \quad \text { Area occupied by effusion at baseline } \% & 39.4 \pm 19.6 & 38.8 \pm 20 & 40.1 \pm 19.4 & 0.76 \\ \quad \text { CT scan } & 86(92 \%) & 47(90 \%) & 39(95 \%) & 0.39 \\ \quad \text { Number of collections } & 2.5 \pm 1.4 & 2.4 \pm 1.4 & 2.6 \pm 1.5 & 0.46 \\ \quad \text { Isolated if } \geqslant 2 \text { collections } & 39(64 \%) & 22(71 \%) & 17(57 \%) & 0.24 \\ \text { Drainage } & & & & \\ \quad \text { Image-guided procedure } & 76(82 \%) & 38(73 \%) & 38(93 \%) & 0.02 \\ \quad \text { Tube size French } & 13.7 \pm 4 & 14.5 \pm 4.8 & 12.6 \pm 2.4 & 0.02 \\ \quad \text { Hospitalisation-to-drainage duration days } & 3(1-8) & 2(0-7) & 3(2-9) & 0.31 \\ \quad \text { Drainage-to-lysis duration days } & 1(0-2) & 1(0-2) & 1(0-2) & 0.23 \\ \end{array}$

Data are presented as mean \pm SD or median (interquartile range), unless otherwise stated. $t-P A$ : tissue plasminogen activator; CT: computed tomography. 


\section{Primary endpoint}

Overall, 34\% of the patients required an additional procedure after an initial fibrinolysis, of whom $21 \%$ received an additional chest tube and 13\% underwent video-assisted thoracic surgery pleural debridement (table 2). Additional chest tubes prevented surgery in $90 \%$ of patients (18 out of 20). No patient underwent a thoracotomy approach. A review of the radiological and biological outcomes confirmed that referrals for surgery or additional drainage were due to the absence of clinical response to the initial fibrinolysis. The difference in the rate of additional procedure between the two groups was not significant $(\mathrm{p}=0.35)$. In a univariate analysis, the need for an additional procedure was predicted by a tube size of 20-24 French as compared to a tube size of 8-12 French (OR 4.86, p=0.02), pleural effusion occupying more than one-third of the hemithorax before fibrinolysis (OR 2.94, $\mathrm{p}=0.04$ ), continuous chest drainage that lasted $>10$ days (OR 6.75, p=0.03) and the presence of multiple pleural collections on CT images (OR 4.81, $\mathrm{p}=0.009$ ) (table 3). However, the type of drainage procedure, the presence of a positive bacterial culture, and the durations from hospitalisation to drainage and from drainage to fibrinolysis were not associated with the need for an additional procedure after initial fibrinolysis (table 3). In a multivariate analysis, the type of fibrinolysis did not predict the need for an additional procedure (OR $0.69, \mathrm{p}=0.48$ ) (table 4). However, the presence of multiple pleural collections (OR 6.58, $\mathrm{p}=0.007$ ) and use of a large-bore drain (OR 11.19, $\mathrm{p}=0.01$ ) could predict it. The risk in performing an additional procedure was $46 \%$ in patients with multiple pleural collections and $15 \%$ in those with a single pleural collection. There was no association between the presence of multiple pleural collections and the quantity of pleural effusion on the chest radiograph at baseline $(\mathrm{p}=0.15)$. When compared with a unique pleural collection, multiple pleural collections were associated with a higher ratio of pleural effusion at day $3(12.9 \pm 3 \%$ versus $24.6 \pm 2.6 \%$, respectively; $\mathrm{p}=0.011$ ). Moreover, the presence of pleural effusion at day 3 was associated with the need for an additional procedure (OR 14.87, 95\% CI 1.88-117.28; $\mathrm{p}=0.01$ ).

In the subgroup analysis, $15 \%$ and $10 \%$ of the patients in the urokinase and t-PA/DNAse groups, respectively, underwent surgery $(\mathrm{p}=0.42)$ (table 2$)$. In the univariate and multivariate analyses, no covariate predicted the chance of surgical referral (tables 3 and 4).

\section{Secondary endpoints}

As shown in table 2, the duration of fibrinolysis was longer among patients who received urokinase than those who received t-PA/DNAse (5 days (IQR 4-7 days) versus 3 days (IQR 3-3 days), respectively; $\mathrm{p}<0.001$ ). However, drainage duration (7 days (IQR 4-10 days) versus 6 days (IQR 5-8 days); $\mathrm{p}=0.05$ ) and length of hospital stay (18 days (IQR 11-25 days) versus 18 days (IQR 11-32 days); $\mathrm{p}=0.12$ ) were not significantly different between the two groups. Complications of fibrinolysis were observed during

\section{TABLE 2 Clinical outcomes}

\begin{tabular}{|c|c|c|c|c|}
\hline Clinical outcomes & Overall & Urokinase & t-PA/DNAse & p-value \\
\hline Additional procedure & $32(34 \%)$ & $20(38 \%)$ & $12(29 \%)$ & 0.35 \\
\hline Surgical referral & $12(13 \%)$ & $8(15 \%)$ & $4(10 \%)$ & 0.42 \\
\hline Additional chest tube & $20(21 \%)$ & $12(23 \%)$ & $8(19 \%)$ & 0.68 \\
\hline Duration of fibrinolysis days & $3(3-5)$ & $5(4-7)$ & $3(3-3)$ & $<0.001$ \\
\hline Duration of drainage days & $6(4-9)$ & $7(4-10)$ & $6(5-8)$ & 0.05 \\
\hline \multicolumn{5}{|c|}{$\begin{array}{l}\text { Change in area occupied by effusion from } \\
\text { baseline \% }\end{array}$} \\
\hline On day 3 & $-19.3 \pm 17.9$ & $-19.4 \pm 16$ & $-19.1 \pm 20.2$ & 0.94 \\
\hline On day 7 & $-28.7 \pm 18$ & $-26.9 \pm 19.2$ & $-30.8 \pm 16.7$ & 0.38 \\
\hline \multicolumn{5}{|c|}{$\begin{array}{l}\text { Change in serum CRP level from baseline } \\
\mathrm{mg} \cdot \mathrm{dL}^{-1}\end{array}$} \\
\hline On day 3 & $-82.3 \pm 86.2$ & $-70.4 \pm 76.1$ & $-96.8 \pm 96$ & 0.15 \\
\hline On day 7 & $-131 \pm 109.2$ & $-104.6 \pm 99.7$ & $-163.7 \pm 112.4$ & 0.01 \\
\hline \multicolumn{5}{|c|}{$\begin{array}{l}\text { Change in blood leukocyte count from } \\
\text { baseline } \times 10^{9} \mathrm{~L}^{-1}\end{array}$} \\
\hline On day 3 & $-2.2 \pm 4.2$ & $-1.6 \pm 4.3$ & $-2.9 \pm 4$ & 0.15 \\
\hline On day 7 & $-3.8 \pm 5.8$ & $-2.1 \pm 5.4$ & $-5.9 \pm 5.5$ & 0.002 \\
\hline Complications of fibrinolysis & $7(8 \%)$ & $0(0 \%)$ & $7(17 \%)$ & 0.002 \\
\hline Length of stay days & $18(11-26)$ & $18(11-25)$ & $18(11-32)$ & 0.12 \\
\hline Death at 30 days & $6(6 \%)$ & $4(8 \%)$ & 2 (5\%) & 0.58 \\
\hline
\end{tabular}

Data are presented as median (interquartile range) or mean \pm SD unless otherwise stated. $t-P A$ : tissue plasminogen activator; CRP: C-reactive protein. 


\begin{tabular}{|c|c|c|c|c|}
\hline \multirow[t]{2}{*}{ Covariate } & \multicolumn{2}{|c|}{ Additional procedure } & \multicolumn{2}{|c|}{ Surgical referral } \\
\hline & OR $(95 \% \mathrm{CI})$ & p-value & OR $(95 \% \mathrm{CI})$ & p-value \\
\hline Urokinase versus t-PA/DNAse & $0.66(0.28-1.59)$ & 0.36 & $0.59(0.17-2.13)$ & 0.43 \\
\hline Age & $1(0.97-1.02)$ & 0.95 & $0.99(0.95-1.02)$ & 0.45 \\
\hline Positive bacterial culture & $1.71(0.71-4.16)$ & 0.23 & $2(0.59-6.79)$ & 0.27 \\
\hline Hospitalisation-to-drainage duration & $0.93(0.85-1.01)$ & 0.07 & $0.87(0.72-1.05)$ & 0.14 \\
\hline Drainage-to-lysis duration & $1.13(0.93-1.36)$ & 0.22 & $1.19(0.96-1.47)$ & 0.12 \\
\hline $\begin{array}{l}\text { Non-image-guided versus image-guided } \\
\text { procedure }\end{array}$ & $2.59(0.89-7.56)$ & 0.08 & $0.88(0.17-4.44)$ & 0.88 \\
\hline \multicolumn{5}{|l|}{ Tube size (ref. 8-12 French) } \\
\hline 14-18 French & $1.06(0.4-2.78)$ & 0.91 & $1.54(0.41-5.79)$ & 0.53 \\
\hline 20-24 French & $4.86(1.26-18.77)$ & 0.02 & $1.72(0.29-10.18)$ & 0.55 \\
\hline Duration of fibrinolysis & $1.18(0.98-1.41)$ & 0.08 & $1.05(0.83-1.33)$ & 0.66 \\
\hline \multicolumn{5}{|l|}{ Duration of drainage (ref. 1-3 days) } \\
\hline 4-10 days & $1.25(0.31-5.1)$ & 0.76 & $0.57(0.1-3.16)$ & 0.52 \\
\hline$>10$ days & $6.75(1.16-39.2)$ & 0.03 & $1.5(0.2-11)$ & 0.69 \\
\hline \multicolumn{5}{|c|}{ Baseline area occupied by effusion (ref. $\leqslant 1 / 3$ ) } \\
\hline $1 / 3$ to $2 / 3$ of the hemithorax & $2.94(1.02-8.5)$ & 0.04 & $1.69(0.4-7.1)$ & 0.47 \\
\hline$>2 / 3$ of the hemithorax & $4.33(1.1-17.1)$ & 0.04 & $1.61(0.24-10.9)$ & 0.49 \\
\hline One versus multiple collections & $4.81(1.48-15.66)$ & 0.009 & $2.53(0.49-11.82)$ & 0.31 \\
\hline Isolated versus grouped collections & $1.27(0.43-3.69)$ & 0.67 & $2.45(0.47-12.78)$ & 0.29 \\
\hline
\end{tabular}

treatment with $\mathrm{t}-\mathrm{PA} / \mathrm{DNAse}$ in seven patients $(\mathrm{p}=0.002)$. All complications corresponded to haemothorax, which was defined by the presence of blood in the drain. In all patients, this bleeding was not associated with a haemodynamic change or a decrease in haemoglobin. The occurrence of haemothorax was not associated with haemostasis disorders. In five patients, the complications required premature termination of fibrinolysis. The management procedure was conservative in all patients, with good clinical outcomes. The mortality rates at 30 days were similar in both groups: four (8\%) and two (5\%) patients had died in the urokinase and t-PA/DNAse groups, respectively $(\mathrm{p}=0.58)$, with lung cancer being the cause of death in all cases.

The levels of pleural effusion, serum CRP and blood leukocytes seemed to decrease over time in a similar fashion in both groups (figure 1); no significant differences were observed in these variables between the two groups at baseline, or on days 3 or 7 . Relative to the corresponding chest radiography findings before fibrinolysis, the changes in the area occupied by the effusion were similar in both groups on days 3 and 7 (table 2). However, the decreases in serum CRP and leukocyte levels between baseline and day 7 were significantly greater in the $\mathrm{t}-\mathrm{PA} / \mathrm{DNase}$ group than the urokinase group $(\mathrm{p}=0.01$ and $\mathrm{p}=0.002$, respectively).

Although the cost per injection of urokinase was lower than that of t-PA/DNAse, the total costs of injections per patient was higher in the urokinase group $(\mathrm{p}=0.04)$ (table 5).

TABLE 4 Odds ratios for failure of fibrinolysis and subgroup surgical referral, determined using a multivariate logistic regression model

\begin{tabular}{|c|c|c|c|c|}
\hline \multirow[t]{2}{*}{ Covariate } & \multicolumn{2}{|c|}{ Additional procedure } & \multicolumn{2}{|c|}{ Surgical referral } \\
\hline & OR $(95 \% \mathrm{CI})$ & $p$-value & OR $(95 \% \mathrm{CI})$ & p-value \\
\hline Urokinase versus t-PA/DNase & $0.69(0.25-1.91)$ & 0.48 & $0.52(0.14-2.02)$ & 0.35 \\
\hline \multicolumn{5}{|l|}{ Tube size (ref. 8-12 French) } \\
\hline 14-18 French & $1.1(0.39-3.14)$ & 0.85 & $1.71(0.44-6.69)$ & 0.44 \\
\hline 20-24 French & $11.19(1.7-73.9)$ & 0.01 & $1.67(0.26-10.78)$ & 0.59 \\
\hline One versus multiple collections & $6.58(1.68-25.73)$ & 0.007 & $2.44(0.48-12.26)$ & 0.28 \\
\hline
\end{tabular}




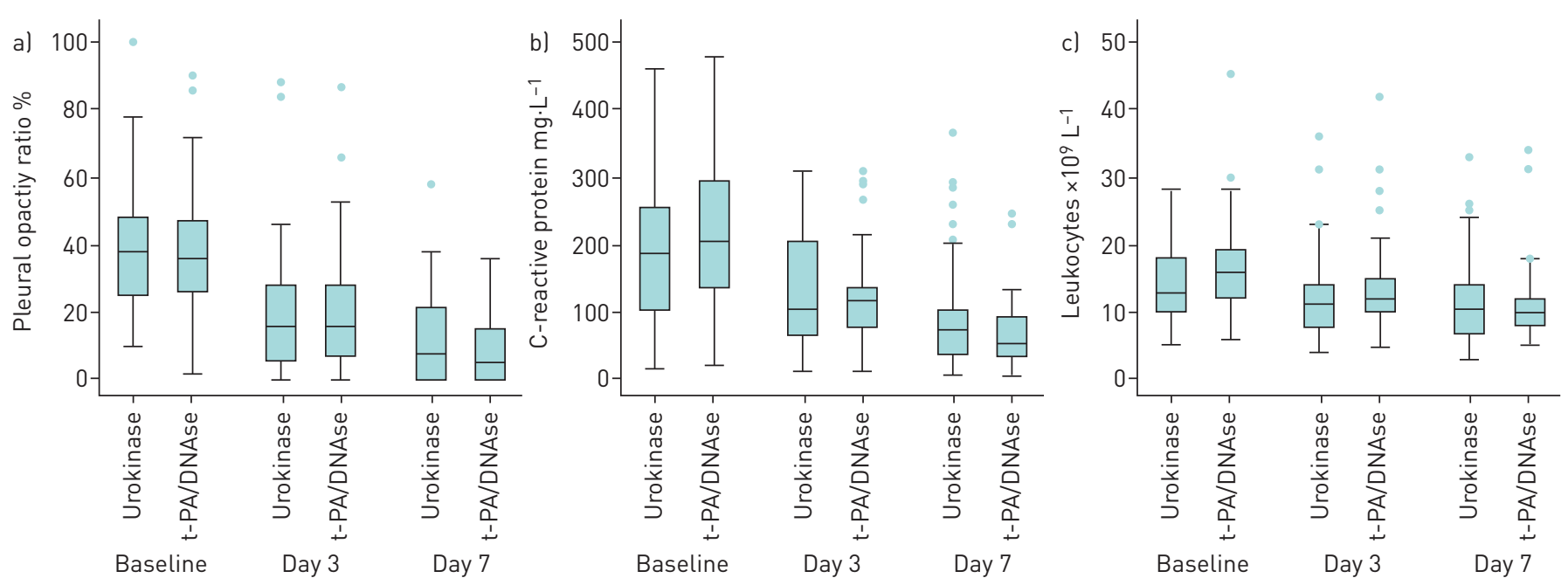

FIGURE 1 Change in a) pleural opacity, b) serum C-reactive protein and c) serum leukocyte counts over time according to the type of fibrinolysis. There were no differences in these variables between groups ( $p>0.05)$. t-PA: tissue plasminogen activator.

\section{Discussion}

Our controlled, prospective cohort study showed that high-concentration urokinase treatment was equally as effective as t-PA/DNAse treatment in patients with pleural infection. The need for an additional chest tube and the rate of surgical referral were similar in both groups. However, the t-PA/DNAse treatment was associated with a higher rate of complications.

In our study, one-third of patients needed an additional procedure after an initial fibrinolysis. There was no difference in the rate of additional procedures between the groups. This high rate of additional procedures was not explained by the median duration of 3 days from hospitalisation to the drainage. Nonetheless, our result is lower than that reported by SEMENKOVICH et al. [9], with a rate of additional procedures of $48 \%$ after an initial chest tube. Additional drainage was the most common procedure and prevented further surgery in most patients. Nevertheless, the rate of surgical referral was low and not significantly different between groups. Our results are similar to those of previous studies, which report rates of surgical referral in urokinase groups (dosage 80000-100000 UI per day) from 4\% to $29 \%$ [7, 10-12]. The rates of surgical referral in t-PA/DNase groups varied from $4 \%$ to $10 \%$ at dosages of $5 \mathrm{mg}$ DNAse and 5-10 mg t-PA [8, 13-16]. The high concentration of urokinase therapy used in this study was developed at our institution. We cannot assess the impact of this high concentration on the success rate of fibrinolysis because of the lack of a control group with the standard urokinase dosage. However, we attest to the safety of this protocol with no complications during fibrinolysis. In contrast, t-PA/DNAse treatment was associated with a high rate of non-serious haemothorax complications, similar to the results in the MIST2 study [8]. However, the presence of blood in the drain could also be due to the breaking down of loculations. In this way, the good clinical course in these patients despite early discontinuation of treatment may be a sign of the effectiveness of fibrinolysis. Nonetheless, in our patients, the presence of blood in the drain required the discontinuation of treatment in order to avoid a significant haemorrhage and subsequent surgery, and is therefore related to a complication. Although the meaning of these haemothoraces is not clearly defined, t-PA/DNAse should be administered cautiously to patients with haemostatic disorders.

Concerning the drainage procedure, our results indicated that large-bore drainage predicts the risk of needing an additional procedure but not referral for surgery. Consequently, the higher rate of large-bore

\section{TABLE 5 Cost of injections}

\begin{tabular}{lccc} 
Cost & Urokinase & t-PA/DNAse & p-value \\
\hline Cost per injection CHF & 144.45 & 229.17 & NA \\
Cost per patient CHF mean \pm sD & $1550.06 \pm 101.92$ & $1263.23 \pm 530.37$ & 0.04 \\
\hline t-PA: tissue plasminogen activator; NA: not applicable. & & \\
\hline
\end{tabular}


drainage in the urokinase group could explain the trend for a higher rate of failure in this group. In our study, a small-bore drain was strongly associated with an imaging-guided procedure. Small-bore catheters are recommended in surgical guidelines, but there has been no superiority trial to support this recommendation [5, 17]. No consensus exists regarding the ideal drainage procedure. Our results demonstrated that further studies are necessary to determine the significance of an imaging-guided procedure in the success of fibrinolysis.

Regarding radiological outcomes, the changes in the area occupied by the effusion between baseline and day 7 were comparable between groups. Interestingly, these results are similar to the significant result observed in the t-PA/DNAse group in the MIST2 study [8]. This confirms the effectiveness of a high concentration of urokinase to drain the pleural fluid. The volume of effusion on the chest radiograph at the baseline did not predict clinical outcome. However, a chest radiograph on day 3 of fibrinolysis appeared useful to estimate the chance of success.

The presence of multiple pleural collections on CT images, isolated or grouped, predicted the necessity of additional procedures in both groups. The type of drainage procedure used in the presence of multiple collections does not explain this result. Furthermore, the rate of image-guided procedures was adequately high in both groups with multiple collections, so the presence of such multiloculated collections suggests the severity of the two cohorts and confirms the utility of lytic therapies. Previous studies have demonstrated high success rates of fibrinolysis in patients with fibrinous septations as shown by ultrasound $[7,13]$. However, further studies are needed to determine the efficacy of fibrinolysis according to the anatomical location of pleural collections and their size.

Regarding biological outcome, the changes in inflammatory marker concentrations by day 7 were more important in the t-PA/DNAse group than the urokinase group, which was most likely to be so due to higher baseline levels in the former group. Nonetheless, this result corresponds to data from the MIST2 study, where the inflammatory marker levels in the t-PA and t-PA/DNAse groups were higher and lower by day 7 , respectively, than the placebo group [8].

The addition of DNAse in the fibrinolysis procedure increased the cost of treatment, which may lead to financial difficulties. In our study, the cost per injection of urokinase was lower than t-PA/DNAse; however, the high dose of urokinase and longer treatment duration made the total cost of this treatment higher than t-PA/DNAse. Further studies, such as that by Piccolo et al. [13], are needed to improve the efficiency of urokinase at a reduced dose.

This monocentric study has some limitations. First, there is some difficulty in interpreting the results due to absence of control group with the use of a placebo. Second, the drainage procedures and duration of fibrinolysis differed significantly between groups. However, age, pleural fluid analysis and radiological findings did not differ significantly between groups. Furthermore, the indication for fibrinolysis was the same for all patients, which could limit any bias. Third, the criteria to perform surgery or insert an additional drain were not clearly protocoled with precise clinical settings, and were dependent on the managing physician and patient's preferences.

Our study shows that urokinase therapy is as effective as t-PA/DNAse therapy in patients with pleural infection, with similar clinical outcomes and fewer complications. Therapy with urokinase or t-PA/DNAse provides satisfactory results and serves as a treatment option for pleural infection. According to our results, we recommend that the drainage of the pleural effusion should be conducted as soon as possible with an imaging-guided, small-bore drain followed by fibrinolysis. The use of a high dose of urokinase should be favoured in patients with haemostatic disorders. The presence of multiple collections should be assessed via CT before performing fibrinolysis. In patients with multiple collections and a persistence of pleural effusion 3 days after the start of fibrinolysis, an additional procedure (surgery or drainage) should be proposed according to the comorbidities of the patient. However, a RCT that compares urokinase and t-PA/DNAse is needed to confirm our results. Furthermore, studies are needed to compare the efficacy of a surgical management as the first-line therapy in comparison with fibrinolysis in patients with multiple collections.

Conflict of interest: B. Bédat has nothing to disclose. J. Plojoux has nothing to disclose. J. Noel has nothing to disclose. A. Morel has nothing to disclose. J. Worley has nothing to disclose. F. Triponez reports fees from MedTronic for organisation of courses (neuromonitoring of the recurrent laryngeal nerve), outside the submitted work. W Karenovics has nothing to disclose.

\section{References}

1 Farjah F, Symons RG, Krishnadasan B, et al. Management of pleural space infections: a population-based analysis. J Thorac Cardiovasc Surg 2007; 133: 346-351. 


\section{Thomson}

empyema. Thorax 2002; 57: 343-347.

11 Tuncozgur B, Ustunsoy H, Sivrikoz MC, et al. Intrapleural urokinase in the management of parapneumonic empyema: a randomized controlled trial. Int J Clin Pract 2001; 55: 658-660.

12 Bouros D, Schiza S, Tzanakis N, et al. Intrapleural urokinase versus normal saline in the treatment of complicated parapneumonic effusions and empyema. A randomized, double-blind study. Am J Respir Crit Care Med 1999; 159: 37-42.

13 Piccolo F, Pitman N, Bhatnagar R, et al. Intrapleural tissue plasminogen activator and deoxyribonuclease for pleural infection: an effective and safe alternative to surgery. Ann Am Thorac Soc 2014; 11: 1419-1425.

14 Majid A, Kheir F, Folch A, et al. Concurrent intrapleural instillation of tissue plasminogen activator and deoxyribonuclease for pleural infection: a single center experience. Ann Am Thorac Soc 2016; 13: 1512-1518.

15 Mehta HJ, Biswas A, Penley AM, et al. Management of intrapleural sepsis with once daily use of tissue plasminogen activator and deoxyribonuclease. Respiration 2016; 91: 101-106.

16 Popowicz N, Bintcliffe O, De Fonseka D, et al. Dose de-escalation of intrapleural tissue plasminogen activator therapy for pleural infection. The alteplase dose assessment for pleural infection therapy project. Ann Am Thorac Soc 2017; 14: 929-936.

17 Rahman NM, Maskell NA, Davies CW, et al. The relationship between chest tube size and clinical outcome in pleural infection. Chest 2010; 137: 536-543. 